\title{
A mixed methods study on the patterns of practice of paediatricians during the COVID-19 pandemic in South India
}

\author{
*K. Shreedhara Avabratha ${ }^{1}$, Riya Mary Tharakan ${ }^{1}$, Sudhir Prabhu $\mathbf{H}^{1}$
}

Sri Lanka Journal of Child Health, 2021; 50(3): 489-494

\begin{abstract}
Objectives: This study assesses the impact of COVID-19 on various aspects of clinical practice including clinic and hospital practice, immunisation and newborn and emergency care services among paediatricians practising in South India. The study also describes the practice of personal protection and risk perception by the same paediatricians
\end{abstract}

Method: Purposive sampling with snowball technique was used. The duration of collection of data was from August to October 2020. Quantitative survey of 176 practising paediatricians from South India was done using a validated questionnaire using Google forms which were sent via email or WhatsApp links. Qualitative data were collected by telephonic interview by the key primary researcher. After obtaining verbal consent, the telephonic conversation was recorded and data was analysed.

Results: While most of the doctors kept their clinics open, with limited timings during the pandemic, $31.5 \%$ completely closed their clinics; $88.8 \%$ have experienced a fall in the number of patients and $92 \%$ experienced a fall in their income. Majority made changes in their clinic practice like using hand sanitizer, minimising bystanders and use of masks and face shields in the clinic. The time spent with each patient during the pandemic was also reduced by $60 \%$. The time spent on counselling sessions has been reduced by $64.3 \%$ of the consultants. Majority of the paediatricians continued to give immunisation but more than half of them experienced a decrease in the number of children brought for immunisation. Among the patients who visited the clinics, majority were for non-respiratory complaints. Most doctors were not involved in paid teleconsultation.

\author{
${ }^{I}$ Father Muller Medical College, Kankanady, \\ Mangalore, India \\ *Correspondence: shreedharkdr@fathermuller.in
}

(iD) https://orcid.org/0000-0002-6903-5047

(Received on 28 March 2021: Accepted after revision on 21 May 2021)

The authors declare that there are no conflicts of interest

Personal funding was used for the project.

Open Access Article published under the Creative

Commons Attribution CC-BY (CC)
Conclusions: COVID 19 has affected paediatricians at all levels. There is a major change in the clinical practice so as to reduce the risk of transmission of the virus.

DOI: http://doi.org/10.4038/sljch.v50i3.9731

(Keywords: COVID 19, paediatricians, practice patterns, pandemic, South India)

\section{Introduction}

The World Health Organization (WHO) declared the novel coronavirus outbreak as a public health emergency of international concern on January 30, $2020^{1}$. The Indian government announced a countrywide lockdown for 3 weeks starting at midnight on March 24 to slow down the spread of COVID-19 as the number of people testing positive in the country reached $563^{2}$. However, this lockdown was further extended till May 3, 2020. One month into lockdown, outpatient clinics and elective surgeries were taking a beating. Effect of the lockdown and the fear of the pandemic among people had resulted in fewer essential healthcare visits, including routine immunization, which could have resulted in outbreak of vaccine preventable diseases $^{3,4}$. In this changed scenario, many patients avoided hospital visits. There is a paucity of scientific evidence regarding the impact of the current pandemic on the healthcare seeking behaviour of the community, especially, the care sought for their children with a paediatrician.

\section{Objectives}

This study assesses the impact of COVID-19 on various aspects of clinical practice including clinic and hospital practice, immunisation, newborn and emergency care services among paediatricians practising in South India. The study also describes the practice of personal protection and risk perception by the same paediatricians.

\section{Method}

Sampling method and sample size: The study used a mixed method approach with quantitative survey of practising paediatricians and a qualitative interview of selected paediatricians using a semistructured approach. Purposive sampling with snowball technique was used. With the assumption of $50 \%$ of study respondents, allowable error of $8 \%$ and non-response rate of $10 \%$, minimum sample size needed was 166 . However we were able to 
achieve a sample size of 176 . Twelve participants from within were selected by convenience sampling by primary researcher for telephonic interview till data saturation was reached. After obtaining verbal consent, the telephonic conversation was recorded and data was analysed.

Study tool: A questionnaire was developed including six sections to collect information on a) socio-demographic profile, b) out-patient clinical practices, c) hospital practices, d) emergency practices, e) delivery and newborn management and f) risk perception and personal protective practices. Questionnaire was prepared based on available literature, considering the scope of objectives and validated by senior paediatricians and pilot testing. Questionnaire was sent to various paediatricians in Southern India via Google forms as emails/WhatsApp links.

Data collection: The duration of collection of data was from August to October 2020. Qualitative data were collected by telephonic interview by the primary researcher.

Inclusion criteria: Paediatricians practising/ working in South India irrespective of whether in the Government or private set up.

Exclusion criteria: Postgraduate students in paediatrics and super-specialists not practising paediatrics.

Ethical issues: Approval was obtained from the Father Muller Institutional Ethics Committee, Father Muller Research Centre, Mangalore, Karnataka, India (No. FMIEC/CCM/411/2020). All care was taken to ensure confidentiality and anonymity. No contact or personal identification details were asked. Only general information was included.
Data analysis: Statistical Package for the Social Sciences Version 21.0 was used to analyse the data. The data were analysed by frequency, percentages and Chi-Square test. Thematic analysis was done for qualitative data.

\section{Results}

A total of 176 doctors gave consent and responded to the questionnaire

General information: The majority $(65.3 \%)$ of respondents were males and $58 \%$ belonged to the age group of $31-40$ years; $67 \%$ of the doctors were post-MD (Doctor of Medicine), 30\% were DCH (Diploma in Child Health), 16.5\% had completed DNB (Diplomate of National Board) and 15\% were super-specialists. The respondents were paediatricians from all states of South India

Work set up: $50 \%$ of them had a private clinic, while the rest did not.

Co-morbidities: $81.8 \%$ of the participants did not have any co-morbidities like diabetes, hypertension or ischaemic heart disease.

Clinic practice: The following results were from 89 paediatricians who have a private clinic practice. Most of the doctors (59.5\%) kept their clinic open, with limited timings, during complete lockdown; $31.5 \%$ of them closed their clinic; $10 \%$ of them continued to keep their clinic open like pre COVID-19 times. Majority of them (88.8\%) had experienced a fall in the number of patients after the pandemic. There was more than 50\% drop in number of cases seen by $15 \%$ of the doctors. The time spent with each patient during the pandemic was reduced by $60 \%$ of the doctors; $58 \%$ of the paediatricians were of the opinion that it was not possible to maintain social distancing in their clinic practice. The changes made by them in their clinic practice are mentioned in Table 1.

Table 1: Changes made in clinic practice $(n=89)$

\begin{tabular}{|l|c|}
\hline \multicolumn{1}{|c|}{ What are the changes you have made in your practice? } & Number (\%) \\
\hline Hand sanitizer for patients and bystanders & $77(86.5)$ \\
\hline Hand sanitizer before and after each patient & $71(79.8)$ \\
\hline N-95 mask & $84(94.4)$ \\
\hline Face shield & $80(89.9)$ \\
\hline Personal Protective Equipment (PPE) & $40(11.2)$ \\
\hline Gloves & $48(49.4)$ \\
\hline Thermal scan before entry & $66(74.9)$ \\
\hline Collecting address and phone number & $72(80.9)$ \\
\hline One bystander allowed & $55(61.8)$ \\
\hline Avoiding throat examination & $31(34.8)$ \\
\hline Avoiding auscultation/auscultating back only & $19(21.3)$ \\
\hline Avoiding palpation & $37(41.6)$ \\
\hline Limit number of patients seen in a day & \\
\hline
\end{tabular}


Notification: Majority of the doctors (52.8\%) notified the Influenza-Like Illness (ILI)/Severe Acute Respiratory Infection (SARI) cases to local health administration every day, whereas others were not consistent with the intimation.

Immunisation: $93.3 \%$ of the doctors continued with immunisation practice post-lockdown. However, more than half of them experienced a decrease in the number of patients for immunisation.

High risk cases: Patients with fever, cough and breathlessness were seen with extra precautions by $64 \%$ of doctors, while $13.5 \%$ of them referred such cases to other hospitals; $22.5 \%$ of them completely avoided seeing such cases in their clinics. Among the patients who visited the clinics, $74 \%$ were for non-respiratory complaints.

Consultation: The consultation charges were mostly kept the same as earlier; however, $16 \%$ of paediatricians increased their charges. Most doctors $(55 \%)$ were not involved in teleconsultation. Among the doctors who had teleconsultation, most $(86 \%)$ did not charge the patients. Display boards and brochures were being used by most of the doctors $(70 \%)$ in their clinic to create awareness about COVID-19. Moreover, paperless documentation had been introduced by $25 \%$ of the doctors with private clinics.

Hospital practice: Majority of the doctors (149) practised in hospitals. Most doctors started frequent hand washing/sanitising in between patients. The most common recommendation given by them to the patients for preventive measures for COVID was to wear masks in public places (99.3\%); 93.3\% advised to maintain physical distancing, $91.3 \%$ advised frequent hand wash, $54.4 \%$ advised to cough into sleeves, $4.7 \%$ advised prophylactic medications.

Intensive care unit (ICU) / Emergency: 115 doctors in the study were handling emergency cases. $53 \%$ of doctors felt that there was no delay in providing emergency care due to COVID-19. Most of them $(41.7 \%)$ were able to attend to emergencies within 5 minutes. In the emergency room, most of them used N-95 masks/respirator (74.8\%), face shield (60\%) and gloves (57.4\%). Fewer doctors (32.2\%) used full Personal Protective Equipment (PPE). Majority of the doctors used nebulisations as earlier (47\%), whereas others used it with special precautions only (46.1\%).

Newborn service: The number of doctors attending newborn deliveries was 115 . Most of the doctors $(56.5 \%)$ attended deliveries in suspected / COVID positive case wearing full PPE, whereas $27.8 \%$ of them attended with only N-95 mask and face shield; $89.6 \%$ of the doctors did not routinely use PPE in the neonatal intensive care unit (NICU). The counselling sessions had been reduced by $64.3 \%$ of the consultants.

\section{Personal level}

$68.8 \%$ of all doctors had restricted their hospital visits and practice after the pandemic. Other factors considered are shown in Table 2.

Table 2: Effect of COVID-19 and lockdown at personal level $(n=176)$

\begin{tabular}{|l|c|}
\hline \multicolumn{1}{|c|}{ Factor } & Number (\%) \\
\hline Time duration of wearing masks at stretch? & $119(67.6)$ \\
$4-6$ hours & $45(25.6)$ \\
$6-8$ hours & $10(05.7)$ \\
12 hours & $02(01.1)$ \\
24 hours & \\
\hline What do you use for hand-washing? & $0(0)$ \\
Only water & $109(61.9)$ \\
Soap and water & $67(38.1)$ \\
Alcohol based sanitizer & $162(92)$ \\
\hline Has your monthly income come down due to the pandemic? & $14(08)$ \\
Yes & $69(39.2)$ \\
No & $64(36.4)$ \\
\hline By what percentage has your income dropped? & $41(23.3)$ \\
$25 \%$ & $02(01.1)$ \\
$50 \%$ & \\
$75 \%$ & $111(63.1)$ \\
$100 \%$ & $42(23.9)$ \\
\hline Has the pandemic had an impact on the time spent with family? & $23(13.1)$ \\
Yes, spending more time & \\
Yes, spending less time & \\
No change & \\
\hline
\end{tabular}


Most of the doctors had not taken hydro-chloroquine (HCQ) prophylaxis (62\%). Most of them relied on journals and webinars as their source for COVID-19 related information $(78 \%$ and $79 \%) ; 64 \%$ of them also relied on WhatsApp /other social media platform for information. Half of them considered themselves to be at medium-risk of contracting the infection, even with all necessary precautions; $30 \%$ of them, on the other hand, considered themselves to be at high-risk.

Vaccine: Majority of them were hopeful that the vaccine will be available by 2021 . They were sceptical about its efficacy, only $15 \%$ of them expected it to be safe for all.

\section{Qualitative analysis}

When asked about the changes in practice made in the clinics, during direct interview, a 33 year old male consultant from Mangalore said "I see patients only by appointments and each patient gets not more than 15 minutes". This was similar to the response given by a 53 year old consultant from Ballari "I restrict myself to only one hospital and also give specific timings for my patients to visit me"

With regard to precautions taken to prevent transmission of the virus, a 45 year old consultant from Mangalore said "Patients are not able to hear me properly because of social distancing norms followed in consultation room and as communication is affected they are not happy and I am also not happy". "Most patients are not happy because of the restrictions that have been set in place, especially in their first visit, but I explain to them and eventually they understand the importance and they oblige" says a 46 year old paediatrician from Bangalore.

The general notion with regard to vaccine was that the vaccine will be available by 2021 . A 45 year old paediatrician said "If with natural infection only, people are getting re-infection, question on efficacy of vaccine will always be there". However, most others had opinions similar to a 38 year old consultant from Kundapur "A vaccine developed will be safe and should have some benefit".

\section{Discussion}

Several safety precautions were made in their clinic practice, and most of them used N-95 masks, hand sanitizers and gloves. In a study done on Paediatricians in Cameron, when they were questioned about the systematic wearing of a mask during consultation, $97 \%$ reported wearing a mask for consultation after the pandemic ${ }^{5}$. They also used disposable surgical masks (96\%), gloves $(80.2 \%)$, hand sanitizers $(99 \%)$, and soap and water $(86.1 \%)^{5}$. Most doctors experienced a fall in the number of patients, similar to the study done among
Paediatricians in Cameron, where the outpatient consultations dropped significantly ${ }^{5}$. In a study done among surgeons, $36.5 \%$ completely stopped outpatient services and $63.5 \%$ had a drop in their services. All elective surgeries were reduced by $93.3 \%$. A survey on ophthalmology practice in India by Nair AG, et al showed that $72.5 \%$ of the practising ophthalmologists in India were in total lockdown ${ }^{7}$. In a recent survey conducted by Irish Dental Association (IDA), it was reported that onefifth of the dentists have closed their practices ${ }^{8}$.

We also found that $92 \%$ of them had a drop in their income, with $60 \%$ of them having a loss of $50 \%$ or more of their usual income. In a similar study done among dentists, around three-fourth of the participants had a financial loss of over $70 \%$ amid the outbreak ${ }^{8}$. A drop of more than $75 \%$ of their monthly income was also reported by $52 \%$ surgeons in a similar study, while $22 \%$ faced $50-75 \%$ reduction $6 ; 62 \%$ mentioned that they were spending more time with family after the pandemic. A systematic review by Shah $\mathrm{S}$, et $a l^{9}$ showed an absence of robust in vivo evidence to support the role of HCQ in prevention of COVID-19. But the National Task Force for COVID-19 recommended HCQ for prophylactic use in asymptomatic healthcare workers involved in the care of suspected or confirmed patients of COVID- $19^{10}$. Of the doctors in our study $62 \%$ did not take HCQ prophylaxis, unlike the findings in the study done among surgeons, where about $52 \%$ of respondents had chemoprophylaxis.

Most of them relied on medical journals and webinars as their source for COVID-19 related information (78\% and $79 \%$ respectively), with $64 \%$ relying also on WhatsApp / other social media platforms. Other survey studies done in this area also show that internet is the main source of information, with $76.1 \%$ utilizing the same, whereas only $5.9 \%$ utilized television and $15.4 \%$ utilized directives given on government/national body sources ${ }^{6}$.

Half of the respondents in our study considered themselves to be at medium risk of contracting infection, even with all necessary precautions, and $30 \%$ of them considered themselves to be at high risk. Even among the ophthalmologists, 59.1\% felt that they were potentially at a higher risk of contracting COVID-19 compared to other specialties while examining patients ${ }^{7}$. Among the paediatricians in another study, concerning the fear of being contaminated, $45.5 \%$ and $19.8 \%$ declared being "very" and "extremely" afraid, respectively. The main reason for that fear was the risk of contaminating their relatives in $85.1 \%$ cases $^{5}$.

A major change seen after the pandemic in other similar studies, was the increase in telemedicine ${ }^{11}$. 
However, most doctors in our study (55\%) were not involved in teleconsultation. This is in contrast to the study among ophthalmologists, where approximately $78 \%(976 / 1260)$ of the respondents had begun telephonic/e-mail/video consultations or consultations over social media applications since the lockdown began ${ }^{7}$. In a similar study among paediatricians in Cameroon, there was a significant increase in the use of telehealth ${ }^{5}$.

Malaysia and the Philippines have even had to postpone their mass polio vaccination campaigns ${ }^{3}$. In our study, $93.3 \%$ of the doctors continued with immunisation practice even post-lockdown. However, more than half of them experienced a decrease in the number of patients for immunisation, including that for pneumococcal and influenza vaccines.

The current study has given a glimpse into the major difficulties faced by paediatricians due to the pandemic and lockdown. This data can help develop a support system for the healthcare workers who are in the frontline. However, there are some limitations. The study is limited only to the doctors in South India. Although we included 176 paediatricians in the study, only 89 of them had a private clinic practice causing a drop in number of response to questions related to the clinic practice. There is scope for a similar study to be done across the nation to have a better understanding of the challenges faced by paediatricians due to the pandemic. There is a need for guidelines regarding safety for future practices and solutions to overcome the financial reductions.

\section{Conclusions}

COVID-19 has affected paediatricians at all levels. There is a major change in the clinical practice to reduce the risk of transmission of the virus. From taking personal protection measures while seeing patients, to reducing the number of patient attenders and time spent for examination and counselling, paediatricians have adapted their practice to the pandemic. Most of them have experienced a fall in their income, but have had more time to spend with their family. Most doctors did not engage in paid teleconsultation, but continued with immunisation even post-lockdown, though the number of patients visiting the hospital had reduced

\section{References}

1. Eurosurveillance Editorial Team. Note from the editors: World Health Organization declares novel coronavirus (2019-nCoV) sixth public health emergency of international concern. Eurosurveillance 2020; 25(5): 200131e https://doi.org/10.2807/15607917.ES.2020 .25.5.200131e
2. Pulla P. Covid-19: India imposes lockdown for 21 days and cases rise. $B M J$ 2020; 368: $\mathrm{m} 1251$

https://doi.org/10.1136/bmj.m1251

PMid: 32217534

3. Hirabayashi K. The impact of COVID-19 on routine vaccinations. Reflections during World Immunization Week 2020. UNICEF/UNI315081/Yuwei. April 2020

4. Santoli JM, Lindley MC, DeSilva MB, et $a l$. Effects of the COVID-19 pandemic on routine paediatric vaccine ordering and administration - United States, 2020. Morbidity and Mortality Weekly Report 2020; 69: 591-3. https://doi.org/10.15585/mmwr.mm6919e 2

PMid: 32407298

5. Enyama D, Chelo D, NoukeuNjinkui J, et al, Impact of the COVID-19 pandemic on paediatricians' clinical activity in Cameroon. Archives de Pédiatrie 2020; 27(8): 423-7.

https://doi.org/10.1016/j.arcped.2020.09.0 04

PMid: 33011025 PMCid: PMC7510582

6. Nasta AM, Goel R, Kanagavel M, et al. Impact of COVID-19 on General Surgical Practice in India. Indian Journal of Surgery 2020; 82: 259-63.

https://doi.org/10.1007/s12262-020-

02443-0

PMid: 32837079 PMCid: PMC7319195

7. Nair AG, Gandhi RA, Natarajan S. Effect of COVID-19 related lockdown on ophthalmic practice and patient care in India: Results of a survey. Indian Journal of Ophthalmology 2020; 68: 725-30. https://doi.org/10.4103/ijo.IJO_1067_20

8. Farooq I, Ali S. COVID-19 outbreak and its monetary implications for dental practices, hospitals and healthcare workers. Postgraduate Medical Journal 2020; 96: 791-2. https://doi.org/10.1136/postgradmedj2020-137781

PMid: 32245754 
9. Shah S, Das S, Jain A, Misra DP, Negi VS. A systematic review of the prophylactic role of chloroquine and hydroxychloroquine in coronavirus disease-19 (COVID-19). International Journal of Rheumatic Diseases 2020; 23(5): 613-9.

https://doi.org/10.1111/1756-185X.13842

PMid: 32281213 PMCid: PMC7262257

10. National Taskforce for COVID-19 Advisory on the use of hydroxychloroquine as prophylaxis for SARSCoV-2 infection. 2020.

https://www.mohfw.gov.in/pdf/Advisoryo ntheuseofHydroxychloroquinasprophylaxi sforSARSCoV2infection.pdf
11. Mahajan V, Singh T, Azad C. Using telemedicine during the COVID-19 pandemic. Indian Pediatrics 2020; 57(7): 658-61.

https://doi.org/10.1007/s13312-020-18956

PMCid: PMC7387251 